\title{
Effects of coping statements on experimental pain in chronic pain patients
}

\author{
This article was published in the following Dove Press journal: \\ Journal of Pain Research \\ 18 August 2009 \\ Number of times this article has been viewed
}

\section{Daniela Roditi \\ Michael E Robinson \\ Nola Litwins \\ Department of Clinical and Health Psychology, University of Florida, Gainesville, FL, USA}

\begin{abstract}
The present study measured the effects of catastrophizing self-statements and positive coping self-statements on cold pressor-induced pain. Participants were 58 adult chronic pain patients with current facial pain. It was hypothesized that catastrophizing would lead to a decrease in pain endurance whereas positive coping would lead to an increase in pain endurance. It was also hypothesized that catastrophizing would lead to an increase in peak pain intensity whereas positive coping would lead to a decrease in peak pain intensity. At pretest, participants submerged their nondominant hand in the cold pressor. Pain sensitivity ranges (PSR) were subsequently determined by calculating the difference between tolerance and threshold times. Ratings of peak pain intensity were measured using a pressure sensitive bladder/transducer Participants underwent random assignment to either a catastrophizing group or a positive coping self-statement group. ANCOVA results revealed that on average, participants employing catastrophizing statements as a coping strategy experienced significantly lower PSR ( $M=35.53$, $\mathrm{SD}=39.71)$ compared to participants employing positive coping self-statements $(\mathrm{M}=73.70$, $\mathrm{SD}=86.14$ ) when controlling for pretest PSR. Group assignment had no significant influence on peak pain intensity ratings. Thus, our results reveal that manipulation of coping causes changes in pain endurance.
\end{abstract}

Keywords: catastrophizing, coping, expectation, pain sensitivity

\section{Introduction}

Response expectancies have been defined in the literature as nonvolitional responses to events. ${ }^{1}$ These are differentiated from Rotter's social learning theory concept of outcome expectancies by virtue of being automatic rather than meditated responses as is the case with outcome expectancies. ${ }^{1,2}$ In addition, response expectancies have been typically differentiated by their virtue of being self-confirming. It has been suggested that response expectancies may be a mechanism by which psychotherapy produces change for the patient. ${ }^{3}$ In particular, Milling and colleagues posited that response expectancies may produce analgesia in pain treatments by creating a cognitive set in which the individual being treated comes to expect reductions in pain. ${ }^{3}$ Therefore, we propose that response expectancies and voluntary cognitive efforts need not be mutually exclusive concepts. It seems as though the cognitive set believed to be responsible for analgesic relief in pain treatments exemplifies this proposition. If a cognitive set is established, it is no longer an automatic, nonvolitional response but rather a deliberate, effortful process. Furthermore, if theory regarding response expectancies is expanded to include both nonvolitional and volitional expectations, then we hold that these can be manipulated by altering existing or creating new cognitive
Correspondence: Michael E Robinson Dept. of Clinical and Health Psychology, PO Box I00I65, University of Florida, Gainesville, FL 32610-0165, USA

$\mathrm{Tel}+\mathrm{I} 3522736617$

Fax +I 3522736156

Emailmerobin@ufl.edu 
sets in which a person expects either diminished pain or increased pain. Therefore, a possible mechanism by which expectancies can be manipulated is via alteration of coping self-statements. Positive self-statements can be induced in participants to produce a change of expectations of a more favorable outcome. Likewise catastrophizing statements may be a mechanism by which expectancies can be negatively manipulated, resulting in pessimistic expectations for a worse outcome.

Catastrophizing is the tendency to exaggerate the negative outcomes of a situation. ${ }^{4}$ More specifically, pain catastrophizing is characterized by pessimistic cognitions leading to exaggerations and negative expectations of the pain experience such as heightened pain perceptions. It involves elements of helplessness and pessimism as captured by the catastrophizing subscale of the Coping Strategies Questionnaire. ${ }^{5}$ Within the aforementioned conceptualization catastrophizing is a type of passive coping. ${ }^{6}$ Catastrophizing has been well-established in the literature as a cognitive mediator in the pain experience and a predictor of the pain experience as indicated by heightened pain sensations and lower thresholds for pain for catastrophizers. ${ }^{7-10}$ Research also suggests a positive correlation between catastrophizing and depression exists in chronic pain patients. ${ }^{411-13}$ Despite the wealth of literature regarding catastrophizing and pain, most research has been correlational, supporting the notion of an antecedent relationship but not a causal one. . $^{9,10,14,15}$

Positive coping self-statements are those that encourage the individual to persist despite pain or to reassess the situation in a more positive light. Relatively few studies have investigated the direct relationship between positive cognitions and pain. Some research has found that higher use of coping self-statements was predictive of higher perceptions of control over pain. ${ }^{16}$ Moreover, it has been suggested that positive coping self-statements interact with pain intensity. ${ }^{17,18}$ There is some indication that positive coping self-statements are correlated with fewer depressive symptoms. ${ }^{19}$

Previous research found that preexisting catastrophizing predicted higher pain intensity in an experimental task on children and adolescents whereas preexisting positive coping self-statements predicted lower pain intensity. ${ }^{20}$ Research by Severeijns and colleagues found that although catastrophizing was successfully manipulated in participants in the experimental group condition it did not moderate the pain experience as neither pain expectancy levels, nor experienced peak pain intensity levels, nor pain tolerance differed significantly from those of participants in the control group condition. ${ }^{21}$ It is important to further investigate the relationship between catastrophizing and pain as well as positive coping and pain using an experimental research design in order to infer a causal relationship between cognitions and pain experience.

The present study aimed to build on the current literature on catastrophizing and pain and positive coping and pain from an experimental perspective by directly manipulating the constructs in a sample of adult chronic pain patients. Most previous studies have not directly manipulated catastrophizing or positive coping and findings from studies that have done so are not consistent with the hypothesized theory. ${ }^{20,21}$ The primary aims were to evaluate the effects of catastrophizing and positive coping on pain sensitivity range (PSR) and peak pain intensity measurements during experimentally induced pain using the cold pressor task. It was hypothesized that catastrophizing would lead to a decrease in pain endurance as measured by PSR and positive coping would lead to an increase in pain endurance as measured by PSR. It was also hypothesized that catastrophizing would lead to an increase in pain intensity measurements whereas positive coping would lead to a decrease in peak pain intensity measurements.

\section{Materials and methods Participants}

Adult chronic pain patients $(\mathrm{n}=58)$ with current facial pain stemming from temporomandibular disorders (TMD) were recruited from the Parker Mahan Facial Pain Center at the University of Florida. Eligibility criteria excluded all those with pain duration shorter than six months, those with pain related to malignant process, those with upper extremity pain, and those with history of severe cardiovascular disease. The mean age of the sample was 39.3 years (range 18-65 years, standard deviation $[\mathrm{SD}]=11.68$ ). Forty-nine participants were female and nine were male. Primary diagnoses included myofascial pain syndrome, bruxism, noxious occlusion, degenerative arthritis, fibromyalgia, and disk displacement. Participants had a mean education level of 13.88 years $(\mathrm{SD}=1.90)$. The mean duration of pain was 97.69 months ( $\mathrm{SD}=95.74)$. The majority of participants were married (38), 15 were single, four were divorced, and one was widowed. The sample was predominantly Caucasian (56); the remaining participants were African American (2).

Participants signed a consent form explaining possible risks associated with the experiment and were all assured that they could withdraw from the study at any time without negative consequences if they chose to. The University of 
Florida Institutional Review Board approved the procedures and protocols of the study.

\section{Apparatuses}

A cold pressor apparatus was used to induce pain in participants. It consisted of a 2.5 cubic foot thermal cooler divided by a fitted screen. One side of the apparatus contained cold water whereas the other side contained ice. Water circulated from one side to the other via a dc bilge pump allowing for water to remain at a constant temperature range of $1-3{ }^{\circ} \mathrm{C}$. The $1-3$ degree variation in the water temperature in the cold pressor is within standard parameters for a cold pressor task.

A pressure sensitive bladder/transducer was used to measure individual ratings of pain intensity in response to the cold pressor apparatus. The pressure sensitive bladder consisted of a blood pressure monitor inflation device linked to a computer via a pressure transducer (an $\mathrm{HC} 11$ micropressor) allowing for analog to digital conversion. The device required $20 \mathrm{lbs}$. of force to reach a maximum voltage of 5 volts. No participants were able to maximize this pressure/voltage, thus eliminating possible confound of ceiling effects. The resolution of the processor allowed for measurement sensitivity to increments of 0.01 volts. Throughout the duration of the tasks, participants were unable to view the computer screen that reported their pain intensity measurements, therefore, precluding participants from altering their pain intensity ratings as a function of knowledge regarding previous ratings.

\section{Materials}

A list of catastrophizing statements was derived from Coping Strategies Questionnaire CSQ-CAT subscale and a list of positive coping statements was created by using statements opposite of the catastrophizing statements derived from the CSQ-CAT subscale. ${ }^{5}$ A list of the catastrophizing selfstatements used is reported in Table 1. A list of the positive coping self-statements used is reported in Table 2.

Table I List of catastrophizing self-statements

This is terrible
This is never going to get better
This is overwhelming
I cannot control the pain
This is worse than I thought
I can't stand it anymore
I feel like I can't go on

\section{Measures}

Pain threshold was determined by the amount of time elapsed from initial immersion of hand into cold pressor apparatus and the moment participants verbally indicated they felt pain.

Pain tolerance was determined by the amount of time elapsed from initial immersion of hand into cold pressor apparatus and the moment participants removed their hand from the cold pressor. A limit of 300 seconds was set for tolerance at which point participants were asked to remove their hand due to risk of injury. A total of ten participants reached the tolerance limit at one or both of the testing phases. No additional pain descriptors were provided or elicited to assist in pain tolerance ratings.

Pain sensitivity ranges were determined for each participant by calculating the difference between tolerance and threshold. PSR was used as a measure of pain endurance and has been found to be a more stable measure of pain than pain tolerance. ${ }^{22}$ This was selected as the dependent variable to preclude bias derived from pre-pain perception often inherent in threshold and tolerance ratings. This allowed us to measure only the time during which participants are experiencing pain at pretest and test phase. Additionally, it enabled us to isolate the time during which participants are concomitantly in pain and actively engaging in a coping response during test phase.

Peak pain intensity was determined by the selecting the highest voltage pain intensity measurement as indicated by the pressure sensitive bladder/transducer.

\section{Methods}

In the pretest, all participants submerged their nondominant hand, palm facing down, in the cold pressor apparatus. Participants were instructed to say "pain" when they first experienced pain (to measure pain threshold) and to keep their hand submerged in the cold water as long as possible (to measure pain tolerance). PSRs were subsequently determined for each participant. Pain intensity measurements

Table 2 List of positive coping self-statements

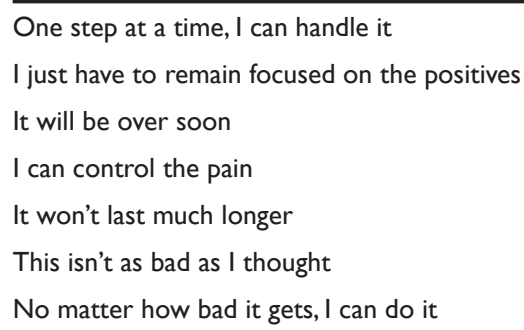


were recorded as indicated by the pressure sensitive bladder/transducer. Participants were instructed to press the pressure sensitive bladder/transducer to indicate the level of pain intensity throughout the duration on the cold pressor task. Only peak pain intensity measurements were used for data analysis. Peak pain intensity measurements were used as opposed to pain intensity measurements at specific time points so as to maximize the number of participants whose data could be used as many participants' data would have been otherwise excluded from the analysis due to very low duration immersion times.

Participants were randomly assigned to one of two groups: Catastrophizing self-statements or positive coping self-statements. Participants in each group were asked to rehearse statements from the designated lists and instructed to choose one statement, repeated aloud, to use as a coping strategy for the duration of the cold pressor task during the test phase. Participants were explicitly asked to repeat the chosen coping strategy aloud during the test phase to ensure treatment integrity, thus enabling researchers to verify that at a minimum some level of the coping cognitive process occurred throughout the test phase cold pressor task.

In the test phase participants repeated the cold pressor task following the same protocol as the pretest with the addition of the chosen coping strategy. Pain threshold and pain tolerance were reassessed. New pain intensity measurements were recorded as indicated by the pressure sensitive bladder/transducer.

Upon completion of the test phase, participants were debriefed concerning the nature of the study. All participants received information regarding appropriate coping strategies in the management of chronic pain.

\section{Results}

In the analyses of our first hypothesis, of the initial 58 participants, only the data from 39 participants were used. Data from the remaining participants were excluded as PSRs could not be calculated because participants either reached the maximized tolerance time of 300 seconds or did not indicate "pain" and, thus, threshold time could not be determined. Among those participants excluded from the analyses, nine were assigned to the catastrophizing group and 10 to the positive self-statements group. Between-group analyses reveal participants did not differ among any of the demographic variables considered (age, gender, race, marital status; $p>0.05)$.

On average, participants employing catastrophizing statements as a coping strategy experienced a 20.05 second decreased PSR (pretest $\mathrm{M}=55.58, \mathrm{SD}=72.45$, test phase $\mathrm{M}=35.53, \mathrm{SD}=39.71$ ) and participants employing positive coping self-statements showed a 12.15 second increase in PSR (pretest $\mathrm{M}=61.55, \mathrm{SD}=87.32$, test phase $\mathrm{M}=73.70$, $\mathrm{SD}=86.14$ ), as shown in Figure 1. To test the effect of coping statement manipulation an ANCOVA with test phase PSR as the dependent variable and pretest PSR as the covariate was performed. Results indicated that after controlling for pre-manipulation PSR, post-manipulation PSR differed between the two coping statement groups. Results indicated a significant effect of coping on PSR after controlling for pretest PSR, $[F(1,36)=5.525 ; p<0.05]$. Manipulation of coping explained $13.3 \%$ of the variance in test phase PSR (see Table 3).

It should be noted that our findings do not reveal any within-group effects. Paired samples $t$-tests indicate that although the positive coping self-statements and the catastrophizing self-statements groups differ significantly from one another at test phase (between-groups effect), each group's endurance at test phase is not significantly different from the mean at pretest $[t(19)=-1.05, p=0.305$, $d=0.33$ and $t(18)=1.54, p=0.140, d=0.59$, respectively] Therefore, the post-intervention difference represents the combination of smaller within-group endurance effects in opposite directions (as hypothesized). The absence of statistically significant within-group differences is likely a function of an underpowered sample size. Nevertheless, $d=0.33$ and $d=0.59$ represent small to medium and medium effect sizes, and suggest these findings might represent meaningful relationships. Of note, there were no significant

\section{Effects of coping manipulation on PSR}

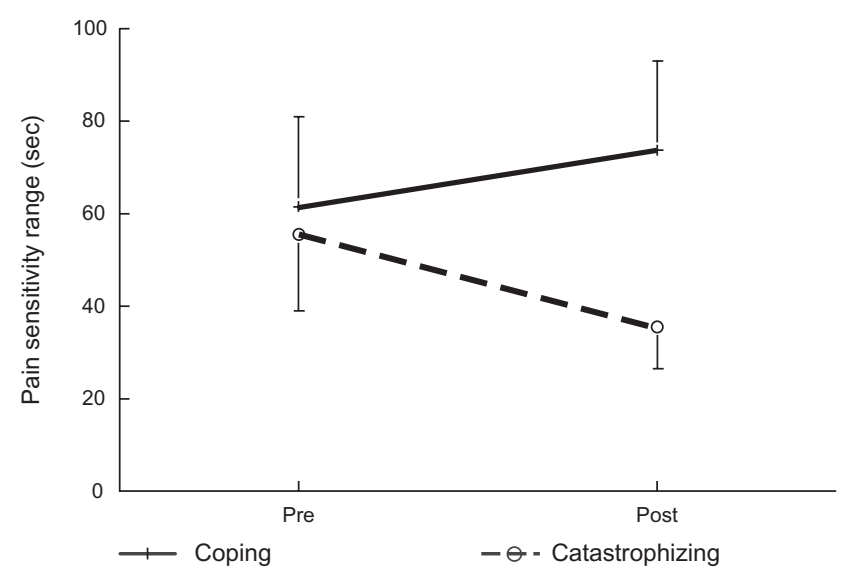

Figure I Mean pain sensitivity ranges (PSR) in seconds. Participants employing catastrophizing statements as a coping strategy experienced decreased PSR from pretest to test phase and participants employing positive coping self-statements showed an increase in PSR from pretest to test phase. 
Table 3 Mean PSR and peak pain intensity measurements of catastrophizing and positive self-statement (PSS) group

\begin{tabular}{llllll}
\hline Group & \multicolumn{2}{l}{ Mean } & & \multicolumn{2}{l}{ Standard deviation } \\
\cline { 2 - 3 } \cline { 5 - 6 } & Pretest & Test phase & & Pretest & Test phase \\
\hline \multirow{2}{*}{ Catastrophizing } & 55.58 & $35.53^{*}$ & 72.45 & 39.71 \\
PSS & 61.55 & $73.70^{*}$ & 87.32 & 86.14 \\
& \multicolumn{2}{l}{ Peak pain intensity } & measurement $(\mathrm{n}=50)$ \\
Catastrophizing & 1.81 & 1.86 & 0.20 & 0.29 \\
PSS & 1.83 & 1.83 & 0.18 & 0.19 \\
\hline
\end{tabular}

Notes: Maximum PSR $=300$ seconds. Maximum peak pain intensity $=5$ volts. Means marked with an asterisk differ at $p<0.05$.

Abbreviations: PSS, positive self-statement; PSR, pain sensitivity ranges.

between-group PSR differences at pretest $[t(45)=-0.065$, $p=0.948]$.

In the analysis of our second hypothesis, of the initial 58 participants, only the data from 50 participants were used. Data from the remaining participants were excluded as no pain intensity measurements were recorded for them.

On average, participants employing catastrophizing statements as a coping strategy did not experience a significant change in test phase peak pain intensity (pretest $\mathrm{M}=1.81$, $\mathrm{SD}=0.20$, test phase $\mathrm{M}=1.86, \mathrm{SD}=0.29$ ) compared to participants employing positive coping self-statements (pretest $\mathrm{M}=1.83, \mathrm{SD}=0.18, \mathrm{M}=1.83, \mathrm{SD}=0.19$ ). To test the effect of coping statement manipulation an ANCOVA with test phase peak pain intensity measurement as the dependent variable and pretest peak pain intensity measurement as the covariate was performed. Results indicated that after controlling for pre-manipulation peak pain intensity measurement, post-manipulation peak pain intensity measurement did not differ significantly between the two coping statement groups. Results indicated a nonsignificant effect of coping on peak pain intensity measurement after controlling for pretest peak pain intensity measurement, $[F(1,47)=0.705, p>0.05]($ see Table 3$)$.

\section{Discussion}

By manipulating the use of coping strategies we were able to evaluate the effects of catastrophizing and positive coping self-statements on pain endurance (PSR) and peak pain intensity during experimentally induced pain. As indicated in the results section, the catastrophizing and positive coping self-statements groups differed post-intervention. Examination of Figure 1 and of the within-group effect sizes suggest the post-interventions differences resulted from smaller nonsignificant within-subjects effects in opposite directions (as hypothesized). The direction of these findings supports extant literature stating catastrophizing is a mediator in the pain experience..$^{8-10,23,24}$ Furthermore, using random assignment and the experimental manipulation of coping statements, results support a causal relationship between coping self-statements and pain perception. Specific evidence suggesting catastrophizing uniquely contributes to decreases in pain endurance is inconclusive. Although several research findings have not found catastrophizing to uniquely affect pain tolerance time there are some research findings supporting this notion. ${ }^{20,21,25}$ It is possible that when several mediators and predictors of pain are evaluated, shared variance (eg, negative affect, fear of pain) eclipses the unique contribution catastrophizing. ${ }^{26,27}$ More work on the unique contributions of negative and positive coping, and negative mood measures is warranted.

Additionally, it is important to highlight that although our results are not applicable to a direct numerical translation to changes in clinical pain endurance, the magnitude of raw change produced by our manipulation is substantial. Specifically, participants in the catastrophizing self-statements group experienced a $56.43 \%$ decrease in endurance ( 20.05 seconds) and those in the positive self-statements group experienced a $16.49 \%$ increased endurance ( 12.15 seconds). We propose that such a change would be of great clinical significance if similar changes were to occur in a clinical setting.

This study adds to the body of literature by furthering the hypothesis of an antecedent relationship between catastrophizing and decreased pain endurance and positive coping. Additionally, it adds to the current body of literature by demonstrating the effects (changes in pain endurance) of experimental manipulation of coping strategies. These findings lend support for this being a potential causal association between coping strategy and pain endurance. It is possible that the use of PSR as a measure of pain endurance is a viable explanation for why we successfully found effects of coping on the pain experience in light of the divergent findings in the extant literature regarding pain tolerance time. ${ }^{20,21,25}$ Whereas other measures of tolerance do not measure the time period of experienced pain and instead measure the time point at which pain becomes unbearable, our measure of endurance includes only the time period during which one is coping with experienced pain by excluding the time period prior to threshold; a subtle but potentially important difference. This explanation would suggest that effects of pain endurance ought to be considered in future research alongside the more commonly used measures of pain threshold and pain tolerance. 
A second aim was to examine the effects of catastrophizing and positive coping on peak pain intensity measurements. Results did not support our hypothesis. The manipulation of coping strategies did not affect peak pain intensity measurements signifying participants did not experience heightened pain upon employing catastrophizing coping strategies nor did they experience a decrease in pain upon employing positive self-statements as coping strategies. Interestingly, although our findings did not support such a relationship, the positive relationship between catastrophizing and heightened pain sensations is widely supported in the extant literature..$^{7-10,28}$ Taken together these findings suggest that the manipulation of coping strategies led participants in our sample to endure pain differentially despite the fact that they were experiencing the same peak pain intensity as they were prior to the manipulation of coping. Interestingly, this lends support for the theory that expectations, rather than demand characteristics, may be the underlying mechanism responsible for the changes in pain endurance. If, indeed, demand characteristics induced by the statements were responsible for the changes observed at test phase then both, pain endurance and pain intensity measurements, should have changed. Instead our results show that the only change was participants' willingness to endure pain regardless of experiencing the same peak intensity of pain. Nevertheless, it is a possibility that demand characteristics motivated participants in the positive coping self-statements group to endure pain for a longer period of time and participants in the catastrophizing self-statements group for a shorter duration of time. Participants, therefore, may have simply been behaving in a manner consistent with the way they believed the experimenter expected them to behave. It remains to be determined if the failure to affect peak pain intensity is a function of the manner in which peak pain was obtained in this study, or if cognitive strategies more strongly operate on more tonic pain, or the endurance of pain. The pressure sensitive bladder/transducer we employed allowed for precise 0.5 second resolution of pain intensity, and unlike other pain intensity measurements, it did not ask participants to rely on retrospective assessment or cumulative averages of their pain in order to rate it. Therefore, it is possible that the absence of a cognitive component in measurement unique to pain may partially account for the lack of coping manipulation effects on peak pain intensity.

Response expectancies have been found to have a significant concordant influence on the pain experience. ${ }^{9,29}$ There is support for the theory that response expectancies partially mediate the relation between catastrophizing and the pain experience and it is likely that response expectancies may also mediate the relation between optimism and the pain experience. ${ }^{9}$ Although there are mixed findings in the literature, optimism has been found to partially mediate the pain intensity in cancer patients and laboratory induced pain. ${ }^{30-32}$ Recent literature reveals that the placebo effect is an example of how expectancies may influence and determine the pain experience. ${ }^{33,34}$ Similarly, we assumed that presenting participants with self-statements of poor pain coping (catastrophizing) or increased pain coping (positive expectations) would generate underlying pain expectations responsible for the changes in PSR. Consistent with the observed effect of catastrophizing and pain in this study, negative expectations have been found to be unique contributors to a heightened pain experience. .,35 $^{9}$ Unfortunately, research exploring positive expectations and their relationship to pain experience is scarce in comparison and findings are often inconclusive. Research has tended to focus predominately on negative contributors to the pain experience almost to the exclusion of positive protective factors in the pain experience. Therefore, although there is some evidence regarding the association between, negative and positive expectations, pessimism and optimism, and decreased pain, additional research in this area is warranted.

\section{Methodological limitations}

The generalizability of the findings in this study is limited to our sample. A design limitation includes the absence of a neutral control group as the pre-post design is subject to pretest sensitization. The absence of a specific manipulation check prevents us from conclusively knowing if our manipulation of coping was responsible for the changes in pain endurance. However, it should be taken into account that participants were instructed to verbalize and repeat the coping strategy they selected aloud throughout the test phase therefore ensuring participants were at least partially cognitively engaging in the particular strategy (via awareness and verbalization). As previous findings in cognitive behavior therapy research suggest, it is a plausible explanation that participants' expectations for pain relief or ability to endure pain changed as a function of the manipulation of coping. ${ }^{36-39}$ Nevertheless, the absence of such a manipulation check tempers a causal inference regarding the effect of changing expectations as the mechanism for change (increased endurance) in the pain experience. Another design limitation is that experimenters were not blinded to the condition or the hypotheses. However, because the data were collected using 
objective time measurements and electronic/mechanical devices it is unlikely this would introduce experimenter bias. Furthermore, it is possible that the cold pressor task was not an optimal analog of chronic clinical pain and that other pain induction strategies would have been more appropriate. Of note, there is no evidence of systematic variance due to variation in water temperature and, thus, no concern of confound effects. As noted in the materials and methods section, this variation is within standard parameters. It should also be noted that no data are available regarding the reliability and validity of the pressure sensitive bladder/transducer used to measure pain intensity. This precludes comparison of our obtained measurements with those obtained from existing well-established methodology for measuring pain intensity such as visual analog scale. Our reliance on PSR as a measure of pain endurance strongly reduced our sample size from 58 to 39, excluding 19 participants for whom PSR could not be calculated. The reduction in sample size may have rendered our study underpowered and may influence the validity of our results. Nonetheless, it should be noted that the exclusion of participants was not selective as the two groups did not differ significantly in between-group analyses of demographic variables. In evaluating our results, it is important to consider the brevity and limited scope of our coping manipulation strategy. Thus, it is suggested that further experimental research be conducted on the effects of catastrophizing and positive coping in the pain experience using more diverse chronic pain samples as well as different pain manipulation strategies. Additional measures of expectation, both situational and dispositional, are important to further understand the potential of expectancies as a mechanism in pain coping strategies, both positive and negative. Lastly, it would be of interest to assess pre-existing coping predispositions prior to pretest and test phase in order to allow for potential associations between baseline coping response predispositions and the effects of experimental manipulation of coping.

\section{Disclosures}

The authors report no conflicts of interest in this work.

\section{References}

1. Kirsch I. Response expectancy as a determinant of experience and behavior. Am Psychol. 1985;40:1189-1202.

2. Rotter JB. Social Learning Theory and Clinical Psychology. Englewood Cliffs, NJ: Prentice Hall; 1954.

3. Milling LS, Reardon JM, Carosella GM. Mediation and moderation of psychological pain treatments: response expectancies and hypnotic suggestibility. J Consult Clin Psychol. 2006;74(2): 253-262.
4. Turner JA, Jensen MP, Romano JM. Do beliefs, coping, and catastrophizing independently predict functioning in patients with chronic pain? Pain. 2000;85(1-2):115-125.

5. Rosenstiel AK, Keefe FJ. The use of coping strategies in chronic low back pain patients: relationship to patient characteristics and current adjustment. Pain. 1983;17(1):33-44.

6. Van Damme S, Crombez G, Eccleston C. Coping with pain: a motivational perspective. Pain. 2008;139(1):1-4.

7. George SZ, Hirsch AT. Psychologic influence on experimental pain sensitivity and clinical pain intensity for patients with shoulder pain. J Pain. 2009;10(3):293-299.

8. Keefe FJ, Lefebvre JC, Egert JR, Affleck G, Sullivan MJ, Caldwell DS The relationship of gender to pain, pain behavior, and disability in osteoarthritis patients: the role of catastrophizing. Pain. 2000;87(3): 325-334.

9. Sullivan MJ, Rodgers WM, Kirsch I. Catastrophizing, depression and expectancies for pain and emotional distress. Pain. 2001;91(1-2): 147-154.

10. Sullivan MJ, Thorn B, Rodgers W, Ward LC. Path model of psychological antecedents to pain experience: experimental and clinical findings. Clin J Pain. 2004;20(3):164-173.

11. Lee EJ, Wu MY, Lee GK, Cheing G, Chan F. Catastrophizing as a cognitive vulnerability factor related to depression in workers' compensation patients with chronic musculoskeletal pain. J Clin Psychol Med Settings. 2008;15(3):182-192.

12. Lopez-Lopez A, Montorio I, Izal M, Velasco L. The role of psychological variables in explaining depression in older people with chronic pain. Aging Ment Health. 2008;12(6):735-745.

13. Sullivan MJ, D'Eon JL. Relation between catastrophizing and depression in chronic pain patients. J Abnorm Psychol. 1990;99(3): 260-263.

14. Roth RS, Lowery JC, Hamill JB. Assessing persistent pain and its relation to affective distress, depressive symptoms, and pain catastrophizing in patients with chronic wounds: a pilot study. Am J Phys Med Rehabil. 2004;83(11):827-834.

15. Severeijns R, Vlaeyen JW, van den Hout MA, Weber WE. Pain catastrophizing predicts pain intensity, disability, and psychological distress independent of the level of physical impairment. Clin J Pain. 2001;17(2):165-172.

16. Haythornthwaite JA, Menefee LA, Heinberg LJ, Clark MR. Pain coping strategies predict perceived control over pain. Pain. 1998;77(1):33-39.

17. Jensen MP, Karoly P. Control beliefs, coping efforts, and adjustment to chronic pain. J Consult Clin Psychol. 1991;59(3):431-438.

18. Jensen MP, Turner JA, Romano JM. Chronic pain coping measures: individual vs composite scores. Pain. 1992;51(3):273-280.

19. Walker LS, Smith CA, Garber J, Claar RL. Testing a model of pain appraisal and coping in children with chronic abdominal pain. Health Psychol. 2005;24(4):364-374.

20. Lu Q, Tsao JC, Myers CD, Kim SC, Zeltzer LK. Coping predictors of children's laboratory-induced pain tolerance, intensity, and unpleasantness. J Pain. 2007;8(9):708-717.

21. Severeijns R, van den Hout MA, Vlaeyen JW. The causal status of pain catastrophizing: an experimental test with healthy participants. Eur $J$ Pain. 2005;9(3):257-265.

22. Wolff BB. Behavioral measurement of human pain. In: Sternbach R, editor. The Psychology of Pain. New York, NY: Raven Press; 1986. p. 155 .

23. Edwards RR, Haythornthwaite JA, Sullivan MJ, Fillingim RB. Catastrophizing as a mediator of sex differences in pain: differential effects for daily pain versus laboratory-induced pain. Pain. 2004;111(3):335-341.

24. Turner JA, Holtzman S, Mancl L. Mediators, moderators, and predictors of therapeutic change in cognitive-behavioral therapy for chronic pain. Pain. 2007;127(3):276-286.

25. France CR, Keefe FJ, Emery CF, et al. Laboratory pain perception and clinical pain in post-menopausal women and age-matched men with osteoarthritis: relationship to pain coping and hormonal status. Pain. 2004;112(3):274-281. 
26. Hirsh AT, George SZ, Bialosky JE, Robinson ME. Fear of pain, pain catastrophizing, and acute pain perception: relative prediction and timing of assessment. J Pain. 2008;9(9):806-812.

27. Hirsh AT, George SZ, Riley JL 3rd, Robinson ME. An evaluation of the measurement of pain catastrophizing by the coping strategies questionnaire. Eur J Pain. 2007;11(1):75-81.

28. Picavet HS, Vlaeyen JW, Schouten JS. Pain catastrophizing and kinesiophobia: predictors of chronic low back pain. Am J Epidemiol. 2002;156(11):1028-1034.

29. Baker SL, Kirsch I. Cognitive mediators of pain perception and tolerance. J Pers Soc Psychol. 1991;61(3):504-510.

30. Allison PJ, Guichard C, Gilain L. A prospective investigation of dispositional optimism as a predictor of health-related quality of life in head and neck cancer patients. Qual Life Res. 2000;9(8):951-960.

31. Kurtz ME, Kurtz JC, Given CW, Given BA. Patient optimism and mastery-do they play a role in cancer patients' management of pain and fatigue? J Pain Symptom Manage. 2008;36(1):1-10.

32. Geers AL, Wellman JA, Helfer SG, Fowler SL, France CR. Dispositional optimism and thoughts of well-being determine sensitivity to an experimental pain task. Ann Behav Med. 2008;36(3):304-313.

33. Vase L, Robinson ME, Verne GN, Price DD. The contributions of suggestion, desire, and expectation to placebo effects in irritable bowel syndrome patients. An empirical investigation. Pain. 2003; 105(1-2):17-25.
34. Pollo A, Amanzio M, Arslanian A, Casadio C, Maggi G, Benedetti F. Response expectancies in placebo analgesia and their clinical relevance. Pain. 2001;93(1):77-84.

35. Montgomery GH, Bovbjerg DH. Presurgery distress and specific response expectancies predict postsurgery outcomes in surgery patients confronting breast cancer. Health Psychol. 2004;23(4):381-387.

36. Smeets RJ, Vlaeyen JW, Kester AD, Knottnerus JA. Reduction of pain catastrophizing mediates the outcome of both physical and cognitive-behavioral treatment in chronic low back pain. J Pain. 2006; 7(4):261-271.

37. Turner JA, Mancl L, Aaron LA. Short- and long-term efficacy of brief cognitive-behavioral therapy for patients with chronic temporomandibular disorder pain: a randomized, controlled trial. Pain. 2006;121(3):181-194.

38. Jensen MP, Turner JA, Romano JM. Changes in beliefs, catastrophizing, and coping are associated with improvement in multidisciplinary pain treatment. J Consult Clin Psychol. 2001;69(4):655-662.

39. Turner JA, Dworkin SF, Mancl L, Huggins KH, Truelove EL. The roles of beliefs, catastrophizing, and coping in the functioning of patients with temporomandibular disorders. Pain. 2001;92(1-2):41-51.
Journal of Pain Research

\section{Publish your work in this journal}

The Journal of Pain Research is an international, peer-reviewed, open access, online journal that welcomes laboratory and clinical findings in the fields of pain research and the prevention and management of pain. Original research, reviews, symposium reports, hypothesis formation and commentaries are all considered for publication.

\section{Dovepress}

The manuscript management system is completely online and includes a very quick and fair peer-review system, which is all easy to use. Visit http://www.dovepress.com/testimonials.php to read real quotes from published authors. 